\title{
Genome-wide in vivo CRISPR screens identify GATOR1 as a TP53 induced tumour suppressor
}

Shinsuke Mizutani ${ }^{1,2,3, \wedge}$, Margaret A Potts ${ }^{1,2, \wedge}$, Yexuan Deng ${ }^{1,2,4}$, Göknur Giner ${ }^{1,2}$, Sarah Diepstraten $^{1,2}$, Andrew J. Kueh ${ }^{1,2}$, Martin Pal ${ }^{1,2,9}$, Geraldine Healey ${ }^{1}$, Lin Tai ${ }^{1}$, Stephen Wilcox $^{1,2}$, Andrew Wei ${ }^{1,6}$, Giovanna Pomilio ${ }^{1,6}$, Yang Liao ${ }^{1,2,5}$, Tony Papenfuss ${ }^{1,2}$, Gemma L. Kelly ${ }^{1,2}$, Wei Shi ${ }^{1,5,7,8}$, Andreas Strasser ${ }^{1,2, *}$, Marco J Herold ${ }^{1,2, *}$

${ }^{1}$ The Walter and Eliza Hall Institute of Medical Research, Melbourne, Australia

2 Department of Medical Biology, University of Melbourne, Melbourne, Australia

${ }^{3}$ Division of Hematology and Oncology, Department of Medicine, Kyoto Prefectural University of Medicine, Kyoto, Japan

${ }^{4}$ The State Key Laboratory of Pharmaceutical Biotechnology, School of Life Sciences, Nanjing University, Nanjing, China

${ }^{5}$ Olivia Newton-John Cancer Research Institute, Heidelberg, Victoria, Australia

${ }^{6}$ Australian Centre for Blood Diseases, Division of Blood Cancers, Monash University, Alfred Medical Research and Education Precinct, Melbourne, Victoria, Australia

${ }^{7}$ School of Computing and Information Systems, The University of Melbourne, Parkville, Victoria 3010, Australia

${ }^{8}$ current affiliation: School of Cancer Medicine, La Trobe University, Heidelberg, Victoria 3084, Australia

${ }^{9}$ current affiliation: School of Dentistry and Medical Sciences, Charles Sturt University, Wagga Wagga, NSW, Australia

${ }^{\wedge}$ These authors contributed equally to this work

* These authors share joint senior authorship 


\begin{abstract}
The tumour suppressor TP53 (called TRP53 in mice) protects cells from neoplastic transformation by activating diverse cellular processes ${ }^{1,2}$. While tumour suppression by TRP53-mediated induction of apoptosis, cell cycle arrest, cell senescence and DNA repair has been well characterised ${ }^{1-4}$, there is so far no example of the loss of a TP53 activated regulator of cellular metabolism promoting tumour development. Using in vivo genomewide CRISPR knockout screens we identified $\mathrm{Nprl3}$ and Depdc5, encoding components of the GATOR1 complex which inhibits mTORC1, as novel tumour suppressors that inhibit c-MYC-driven lymphomagenesis. In a parallel in vivo CRISPR knockout screen using a focused library of sgRNAs targeting TRP53 binding sites in gene promoters/enhancers, we discovered that loss of a TRP53 binding site in the Nprl3 promoter accelerated c-MYC-driven lymphomagenesis to a similar extent as loss of the Nprl3 gene itself. These findings along with the observations that (i) Nprl3 is upregulated in response to DNA damaging drugs in wild-type (wt) but not TRP53 knockout cells and that (ii) GATOR1 deficient $E \mu$-Myc lymphomas all retained wt TRP53 function, whereas $\sim 30 \%$ of control E $\mu$-Myc lymphomas had selected for mutations in Trp53, suggested that direct transcriptional inducton of GATOR1 by TRP53 is a critical tumour suppressive mechanism. Additionally, we found that GATOR1 deficient tumours exhibited abnormally increased mTORC1 signalling, which rendered them highly sensitive to the mTORC1 inhibitor rapamycin, both in vitro and in vivo. Collectively, these findings identify the first mechanism by which TRP53 suppresses tumourigenesis by transcriptional activation of a regulator of metabolism and they also reveal a potential bio-marker to predict responses to mTORC1 inhibitors in the clinic.
\end{abstract}

\title{
Main
}

The transcription factor TP53/TRP53 is critical for tumour suppression and its gene is mutated in $\sim 50 \%$ of human cancers ${ }^{1,2,5}$. Diverse cellular stresses, including those induced by oncogenes, lead to the activation of TRP53, which then controls the transcription of several hundred target genes directly and indirectly, that coordinate a multitude of cellular responses. These include induction of apoptotic cell death, cell cycle arrest/cell senescence as wells as coordination of DNA repair and adaptation of metabolism (Warburg effect) ${ }^{1,2,5}$. It is still debated which of these cellular responses is most critical 
for TRP53-mediated tumour suppression, and this may likely vary depending on the type of cell undergoing neoplastic transformation and the nature of the oncogenic lesion(s) driving tumorigenesis ${ }^{1,2,5}$. While the loss of TRP53 target genes involved in apoptosis (e.g. Puma) ${ }^{6,7}$ or DNA repair (e.g. Mlhl) ${ }^{4}$ were shown to promote oncogene induced tumourigenesis, this has so far not been reported for any TRP53 target gene involved in other cellular processes, such as metabolism. We have conducted in primary cells in vivo genome-wide CRISPR/Cas9 knockout screens to identify the tumour suppressor mechanisms in MYC-driven lymphomagenesis. These experiments were complemented with in vivo CRISPR/Cas9 screens in primary cells using focused libraries containing sgRNAs targeting TRP53 binding sites in promoters/enhancers of TRP53 target genes of interest $^{8-10}$.

\section{Genome wide in vivo CRISPR knockout screen identifies novel tumour suppressor genes}

To identify novel tumour suppressor genes, we conducted whole genome and focused library CRISPR screens in primary cells in vivo using a model of c-MYC-driven lymphomagenesis (Fig.1a) ${ }^{11}$. These screens are likely to identify critical effectors of TRP53-mediated tumour suppression because $\sim 20 \%$ of lymphomas arising in E $\mu$-Myc transgenic mice are selected for mutations in $\operatorname{Trp} 53^{12}$ and loss of even a single allele of Trp53 or its pro-apoptotic target gene, Puma, substantially accelerates lymphomagenesis in $E \mu$-Myc transgenic mice ${ }^{13,14}$. We transduced haematopoietic stem/progenitor cells (HSPCs) from foetal livers of E13.5 E $\mu$-Myc_Cas9 double transgenic mouse embryos (C57BL/6-Ly5.2 background) with a pooled whole genome sgRNA library containing 87,987 sgRNAs targeting 19,150 mouse protein coding genes (4-5 sgRNAs per gene) ${ }^{15}$. As a positive control, we used a sgRNA targeting mouse Trp53 that is known to accelerate c-MYC-driven lymphoma development ${ }^{16}$. As a negative control, we used a sgRNA targeting the human BIM gene (hereafter called control sgRNA). The sgRNA transduced $E \mu-M y c \_C a s 9$ transgenic HSPCs were transplanted into lethally irradiated C57BL/6Ly5.1 mice (Fig. 1a). Mice reconstituted with E $\mu$-Myc_Cas9 HSPCs that had been transduced with the negative control sgRNA developed lymphoma with a median latency of $\sim 140$ days post-transplantation (Fig. 1b). Consistent with previous observations ${ }^{17}$, the mouse Trp53 sgRNA greatly accelerated lymphoma development (median latency 25 days; Fig. 1b). Notably, some mice that had been transplanted with $E \mu-M y c \_C a s 9$ HSPCs 
transduced with the whole mouse genome sgRNA library showed more rapid lymphoma development (median latency $\sim 74$ days) compared to the negative control sgRNA cohort (Fig. 1b). All of the reconstituted mice, regardless of the sgRNA that had been transduced into the donor HSPCs, developed $\operatorname{sIg}^{-}$pre-B or $\operatorname{sig}^{+}$B cell lymphomas (Extended Data Fig.1a, b).

Lymphomas that arose in mice reconstituted with whole genome sgRNA library transduced E $E$-Myc_Cas 9 HSPCs prior to 75 days post-transplantation were deemed accelerated and further investigated ( $n=60 / 113$ sgRNA library recipient mice). Genomic DNA was extracted from the spleen of sick mice and the sgRNAs identified by next generation sequencing (NGS). Most of the tissue sample used for DNA extraction consisted of malignant lymphoma cells in which we identified a small number of highly enriched sgRNAs. However, we also detected at low read counts a large number of sgRNAs, collectively targeting $>75 \%$ of all genes. We speculate that these sgRNAs were present in the non-malignant haematpoietic cells of the sample (Extended Data Fig 1c-d). This indicates a very high representation of the genome-wide library in the transduced $E \mu-M y c \_C a s 9$ HSPCs used for the screen, where only a small number were able to accelerate c-MYC-driven lymphomagenesis. The hits from this screen were prioritised using the following criteria: (1) the sgRNA was within the top 4 enriched sgRNAs within a given lymphoma, (2) the sgRNA was within the top 4 enriched sgRNAs in two or more independent lymphomas, (3) more than one sgRNA targeting the same gene was enriched in two or more lymphomas (Extended Data Table 1). Amongst the top hits were several known tumour suppressors, with the most prominent hit being Trp53, for which all four sgRNAs in the library were enriched across 14 different lymphomas (Extended Data Fig 1e). sgRNAs targeting $T s c 1$ and $T s c 2$ and three other known tumour suppressor genes were also identified (Extended Data Fig 1e). Notably, the screen also identified new candidate tumour suppressors, including Nprl3 (two sgRNAs enriched across 3 lymphomas; Fig 1c) and Depdc5 (one sgRNA enriched in one tumour; Extended Data Fig 1e). Nprl3 and Depdc5 both encode components of the GATOR1 complex, a negative regulator of mTORC1, emphasising the importance of this complex in tumour suppression.

\section{Components of the GATOR1 complex function as tumour suppressors}


To validate NPRL3 and DEPDC5 as tumour suppressors, two independent sgRNAs for each of these genes were individually introduced into E $\mu$-Myc_Cas 9 HSPCs to test whether the loss of these genes was able to accelerate c-MYC-driven lymphomagenesis in reconstituted mice. Notably, all sgRNAs targeting either Nprl3 or Depdc5 tested, accelerated c-MYC-induced lymphomagenesis to a similar extent as the Trp53 sgRNA (Fig. 2a-b, Extended Data Fig 2a-f). Lymphomas with defects in the TRP53 pathway show high levels of mutant TRP53 protein and/or p19/ARF 3,12,18. Consistent with previous reports 3,12 , immunoblotting for TRP53 and p19/ARF revealed that $\sim 30 \%$ of control lymphomas exhibited TRP53 pathway aberrations, but of note, no such abnormalities were found in the NPRL3 or DEPDC5 deficient lymphomas (Fig. 2c-d). This suggests that in mouse lymphomas, defects in the GATOR1 complex obviate the need to acquire mutations in Trp53. Interestingly, human TCGA Pan-Cancer analysis revealed in tumours expressing high levels of $M Y C$ (top 25\%) that low mRNA expression of GATOR1 complex components (bottom 25\%) correlates with significantly poorer survival (median survival 56 months) compared to high GATOR1 mRNA expression (top $25 \%$ ) (median survival 70 months, $P<0.0001$; Fig 2e). These findings demonstrate that NPRL3 and DEPDC5 can function as tumour suppressors in c-MYC-driven lymphomagenesis.

\section{Mutations in the TRP53 binding site of the $\mathrm{Nprl3}$ gene promoter accelerate c- MYC-driven lymphomagenesis}

Since sgRNAs targeting Nprl3 or Depdc5 accelerated c-MYC-driven lymphoma development to a similar extent as sgRNAs targeting Trp53 and since no TRP53 pathway aberrations were found in the NPRL3 or DEPDC5 deficient lymphomas, we considered whether these genes were directly or indirectly regulated by TRP53. To address this question and to more broadly screen for TRP53 target genes that restrain c-MYC-driven lymphomagenesis, we designed a focused sgRNA library, targeting TRP53 binding sites in promoters/enhancers of known TRP53 target genes ${ }^{3,10,19}$. This library contains 1,548 sgRNAs targeting 751 unique gene promoter or enhancer elements that had been discovered by TRP53 ChIP sequence analysis in primary mouse B lymphocytes ${ }^{10,19}$ (Fig. $3 \mathrm{a}, \mathrm{b})$. We transduced $E \mu$-Myc_Cas9 HSPCs with the TRP53 enhancer/promoter focused sgRNA library, again using a sgRNA targeting Trp53 as a positive control and a negative 
control sgRNA. Interestingly, two of the top sgRNAs identified in accelerated lymphomas (mice sick prior to 75 days post-transplantation with sgRNA library transduced $E \mu-M y c \_C a s 9$ HSPCs) were found to target a TRP53 binding site within the promoter region of the Nprl3 gene (Fig. 3c-d, Extended Data Fig 3). Validation experiments using this sgRNAs individually in E $E$-Myc_Cas 9 HSPCs, confirmed that targeting this TRP53 binding site within the Nprl3 promoter significantly accelerated cMYC-driven lymphoma development (median latency $\sim 18$ days; control sgRNA 196 days, $P<0.0001)$, remarkably, to an extent comparable to the sgRNAs targeting Trp53 (median latency $\sim 21$ days, $P<0.0001$ vs control sgRNA) (Fig 3d). To further assess whether TRP53 regulates $N p r l 3$, isogenic $E \mu-M y c$ lymphoma cell lines deficient (Trp53 KO) or sufficient (Trp53 wt) for TRP53 were treated with etoposide, a DNA damaging agent that activates TRP53 ${ }^{2}$. As anticipated, Cdkn1 (p21) expression, a well-known TRP53 target gene, increased following treatment with etoposide in the Trp53 wt lymphomas lines but not in their Trp53 KO derivatives (Fig 3e). Nprl3 expression increased $\sim 2$-fold upon treatment with etoposide in the Trp53 wt lines lines but not in their Trp53 KO derivatives (Fig 3e). These findings functionally validate previous ChIP findings by revealing direct transcriptional activation of Nprl3 through TRP53 constituting a critical tumour suppressive mechanism in c-MYC-driven lymphomagenesis ${ }^{10}$.

\section{GATOR1 deficient lymphomas are addicted to mTORC1 signalling}

Since NPRL3 and DEPDC5 are critical components of the GATOR1 complex that inhibits mTORC1 ${ }^{9}$, we hypothesised that $E \mu$-Myc lymphomas with loss of these proteins would be "addicted" to high levels of mTORC1 signalling. Analysis of mTORC1 activity by intracellular staining for phosphorylated (i.e. activated) S6 kinase (phospho-S6K) revealed that, at steady state, NPRL3 and DEDPC5 deficient lymphoma cells exhibited a large increase in mTORC1 activity compared to control lymphoma cells (Fig. 4a-b). Treatment with rapamycin, a direct inhibitor of mTORC1, markedly reduced the levels of phospho-S6 kinase in NPRL3 as well as DEPDC5 deficient lymphomas to the levels seen in control lymphomas (Fig. 4a, Extended data Fig 3a-c). Consequently, the lymphomas whose development had been accelerated by sgRNAs targeting Nprl3 or Depdc5 were highly sensitive to the $\mathrm{mTORC} 1$ inhibitors rapamycin and torin 1 in vitro (Fig. 4b-c). Remarkably, mice transplanted with NPRL3 or DEPDC5 deficient 
lymphomas could be cured by treatment with the mTORC1 inhibitor rapamycin, whereas control lymphomas did not respond to this treatment (Fig. 4d). These results demonstrate that c-MYC-driven lymphomas whose development was accelerated by the loss of NPRL3 or DEPDC5 are addicted to high levels of mTORC1 signalling and therefore highly sensitive to mTOR inhibitors.

\section{Discussion}

CRISPR gene knockout screens are a powerful approach to identify critical regulators of biological processes of interest ${ }^{20,21}$. While in vivo CRISPR screens have been conducted in mice, they either involved cancer cell lines which are easy to expand and transduce with sgRNA libraries ${ }^{22}$, or transduction of primary cells with only small focussed sgRNA libraries ${ }^{23}$. To our knowledge, the screen presented here is the first whole genome sgRNA library screen conducted in vivo using primary cells, in this case HSPCs from foetal livers of $E \mu$-Myc_Cas 9 embryos. Our work demonstrates that whole genome in vivo CRISPR gene knockout screens in primary cells were able to identify genes that suppress tumorigenesis driven by deregulated c-MYC expression, which is found in $\sim 70 \%$ of human cancers ${ }^{24}$. This success indicates that this approach can also be applied to other biological questions, such as identifying novel suppressors of tumorigenesis driven by different oncogenic lesions, perhaps also in non-haematopoietic cell types, as long as they are amenable to sgRNA library transduction and transplantation into the relevant tissue (e.g. transduction of mammary epithelial stem cells with a sgRNA library followed by their transplanatation into cleared mammary fat pads of mice).

Our screen and subsequent validation studies identified GATOR1 mediated inhibition of mTORC1 as a novel tumour suppressive pathway induced by TRP53. Several TRP53 target genes implicated in the control of metabolism, such as TIGAR, have been identified through gene expression analysis in cancer cell lines ${ }^{25}$. However, thus far there are no reports that loss or mutation of a TRP53 target gene implicated in the control of metabolism can promote tumorigenesis. The explanation for this may be that many genes that are highly up-regulated in response to the activation of TRP53 are not necessarily critical for tumour suppression ${ }^{26-28}$. Of note, to recapitulate the cancer predisposition caused by loss of TRP53 ${ }^{29,30}$, often several TRP53 target genes, such as Puma, p21 and Zmat 3 must all be inactivated simultaneously ${ }^{4,31}$, due to the extensive mutual back-up 
for tumour suppression provided by the diverse cellular responses that can be activated by TRP53.

Here, by employing the highly efficient CRISPR/Cas9 knockout system in primary cells using both an unbiased genome-wide sgRNA library and a focussed library containing sgRNAs that target published TRP53 binding sites in promoters/enhancers of TRP53 target genes we identify the first TRP53-mediated regulation of metabolic processes that is critical for tumour suppression, at least in the context of de-regulated expression of cMYC. We show that direct transcriptional activation of a gene, Nprl3, encoding a component of the GATOR1 complex that inhibits mTORC1 inhibits c-MYC-driven lymphomagenesis. Accordingly, low levels of GATOR1 are predictive of poorer survival in patients with tumours expressing high levels of $c-M Y C$, indicating that this process may also be critical in human cancers and diverse types of tumours. Of note, our work also revealed that enhanced lymphomagenesis caused by defects in the GATOR1 complex engenders a vulnerability to mTORC1 inhibitors that may be used as a biomarker for such agents in clinical cancer trials. 


\section{References}

1 Kastenhuber, E. R. \& Lowe, S. W. Putting p53 in Context. Cell 170, 1062-1078, doi:10.1016/j.cell.2017.08.028 (2017).

2 Vousden, K. H. \& Lane, D. P. p53 in health and disease. Nat Rev Mol Cell Biol 8, 275-283, doi:10.1038/nrm2147 (2007).

3 Michalak, E. M. et al. Puma and to a lesser extent Noxa are suppressors of Mycinduced lymphomagenesis. Cell Death Differ 16, 684-696 (2009).

Janic, A. et al. DNA repair processes are critical mediators of p53-dependent tumor suppression. Nat Med 24, 947-953, doi:10.1038/s41591-018-0043-5 (2018).

5 Levine, A. J., Momand, J. \& Finlay, C. A. The p53 tumour suppressor gene. Nature 351, 453-456 (1991).

6 Villunger, A. et al. p53- and drug-induced apoptotic responses mediated by BH3only proteins puma and noxa. Science 302, 1036-1038, doi:10.1126/science.1090072 (2003).

7 Jeffers, J. R. et al. Puma is an essential mediator of p53-dependent and independent apoptotic pathways. Cancer Cell 4, 321-328 (2003).

8 Bar-Peled, L. et al. A Tumor suppressor complex with GAP activity for the Rag GTPases that signal amino acid sufficiency to mTORC1. Science 340, 1100-1106, doi:10.1126/science.1232044 (2013).

9 Saxton, R. A. \& Sabatini, D. M. mTOR Signaling in Growth, Metabolism, and Disease. Cell 168, 960-976, doi:10.1016/j.cell.2017.02.004 (2017).

10 Tonelli, C., Amati, B. \& Morelli, M. J. p53 transcriptional programs in B cells upon exposure to genotoxic stress in vivo: Computational analysis of nextgeneration sequencing data. Genomics Data 7, 29-31, doi:https://doi.org/10.1016/j.gdata.2015.11.006 (2016).

11 Adams, J. M. et al. The c-myc oncogene driven by immunoglobulin enhancers induces lymphoid malignancy in transgenic mice. Nature 318, 533-538, doi:10.1038/318533a0 (1985).

12 Eischen, C. M., Weber, J. D., Roussel, M. F., Sherr, C. J. \& Cleveland, J. L. Disruption of the ARF-Mdm2-p53 tumor suppressor pathway in Myc-induced lymphomagenesis. Genes Dev 13, 2658-2669 (1999).

13 Hemann, M. T. et al. Suppression of tumorigenesis by the p53 target PUMA. Proceedings of the National Academy of Sciences of the United States of America 
101, 9333-9338 (2004).

14 Michalak, E. M., Villunger, A., Adams, J. M. \& Strasser, A. In several cell types the tumour suppressor p53 induces apoptosis largely via Puma but Noxa can contribute. Cell Death Differ 15, 1019-1029 (2008).

15 Koike-Yusa, H., Li, Y., Tan, E. P., Velasco-Herrera, M. D. C. \& Yusa, K. Genomewide recessive genetic screening in mammalian cells with a lentiviral CRISPRguide RNA library. Nature Biotechnology 32, 267-273, doi:10.1038/nbt.2800 (2014).

16 Aubrey, B. J. et al. An inducible lentiviral guide RNA platform enables the identification of tumor-essential genes and tumor-promoting mutations in vivo. Cell Rep 10, 1422-1432, doi:10.1016/j.celrep.2015.02.002 (2015).

17 Aubrey, B. J. et al. Mutant TRP53 exerts a target gene-selective dominantnegative effect to drive tumor development. Genes Dev 32, 1420-1429, doi:10.1101/gad.314286.118 (2018).

18 Schmitt, C. A., McCurrach, M. E., de Stanchina, E., Wallace-Brodeur, R. R. \& Lowe, S. W. INK4a/ARF mutations accelerate lymphomagenesis and promote chemoresistance by disabling p53. Genes Dev 13, 2670-2677 (1999).

19 Korkmaz, G. et al. Functional genetic screens for enhancer elements in the human genome using CRISPR-Cas9. Nat Biotechnol 34, 192-198, doi:10.1038/nbt.3450 (2016).

20 Shalem, O. et al. Genome-scale CRISPR-Cas9 knockout screening in human cells. Science 343, 84-87, doi:10.1126/science.1247005 (2014).

21 Wang, T., Wei, J. J., Sabatini, D. M. \& Lander, E. S. Genetic Screens in Human Cells Using the CRISPR-Cas9 System. Science 343, 80-84, doi:doi:10.1126/science.1246981 (2014).

22 Chen, S. et al. Genome-wide CRISPR screen in a mouse model of tumor growth and metastasis. Cell 160, 1246-1260, doi:10.1016/j.cell.2015.02.038 (2015).

23 Ye, L. et al. In vivo CRISPR screening in CD8 T cells with AAV-Sleeping Beauty hybrid vectors identifies membrane targets for improving immunotherapy for glioblastoma. Nat Biotechnol 37, 1302-1313, doi:10.1038/s41587-019-0246-4 (2019).

24 Pelengaris, S., Khan, M. \& Evan, G. c-MYC: more than just a matter of life and death. Nat Rev Cancer 2, 764-776, doi:10.1038/nrc904 (2002). 
25 Bensaad, K. et al. TIGAR, a p53-Inducible Regulator of Glycolysis and Apoptosis. Cell 126, 107-120, doi:https://doi.org/10.1016/j.cell.2006.05.036 (2006).

26 Brady, C. A. et al. Distinct p53 transcriptional programs dictate acute DNAdamage responses and tumor suppression. Cell 145, 571-583, doi:10.1016/j.cell.2011.03.035 (2011).

27 Valente, L. J. et al. p53 efficiently suppresses tumor development in the complete absence of its cell-cycle inhibitory and proapoptotic effectors p21, Puma, and Noxa. Cell Rep 3, 1339-1345, doi:10.1016/j.celrep.2013.04.012 (2013).

$28 \mathrm{Li}, \mathrm{T}$. et al. Tumor suppression in the absence of p53-mediated cell-cycle arrest, apoptosis, and senescence. Cell 149, 1269-1283, doi:10.1016/j.cell.2012.04.026 (2012).

29 Donehower, L. A. et al. Mice deficient for p53 are developmentally normal but susceptible to spontaneous tumours. Nature 356, 215-221, doi:10.1038/356215a0 (1992).

30 Jacks, T. et al. Tumor spectrum analysis in p53-mutant mice. Curr Biol 4, 1-7 (1994).

31 Bieging-Rolett, K. T. et al. Zmat3 Is a Key Splicing Regulator in the p53 Tumor Suppression Program. Mol Cell 80, 452-469.e459, doi:10.1016/j.molcel.2020.10.022 (2020).

32 Herold, M. J., van den Brandt, J., Seibler, J. \& Reichardt, H. M. Inducible and reversible gene silencing by stable integration of an shRNA-encoding lentivirus in transgenic rats. Proceedings of the National Academy of Sciences of the United States of America 105, 18507-18512, doi:10.1073/pnas.0806213105 (2008).

33 Chu, V. T. et al. Efficient generation of Rosa26 knock-in mice using CRISPR/Cas9 in C57BL/6 zygotes. BMC Biotechnology 16, 4, doi:10.1186/s12896-016-0234-4 (2016).

34 Kelly, G. L. et al. Targeting of MCL-1 kills MYC-driven mouse and human lymphomas even when they bear mutations in p53. Genes Dev 28, 58-70, doi:10.1101/gad.232009.113 (2014).

35 Cerami, E. et al. The cBio cancer genomics portal: an open platform for exploring multidimensional cancer genomics data. Cancer Discov 2, 401-404, doi:10.1158/2159-8290.Cd-12-0095 (2012).

36 Gao, J. et al. Integrative analysis of complex cancer genomics and clinical profiles 
bioRxiv preprint doi: https://doi.org/10.1101/2022.02.16.480657; this version posted February $17,2022$. The copyright holder for this

preprint (which was not certified by peer review) is the author/funder, who has granted bioRxiv a license to display the preprint in perpetuity. It is made available under aCC-BY-ND 4.0 International license.

using the cBioPortal. Sci Signal 6, pl1, doi:10.1126/scisignal.2004088 (2013). 
a

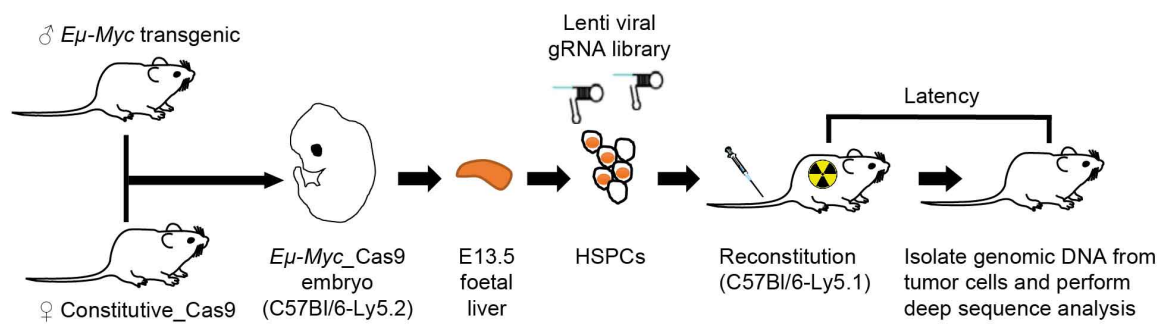

b

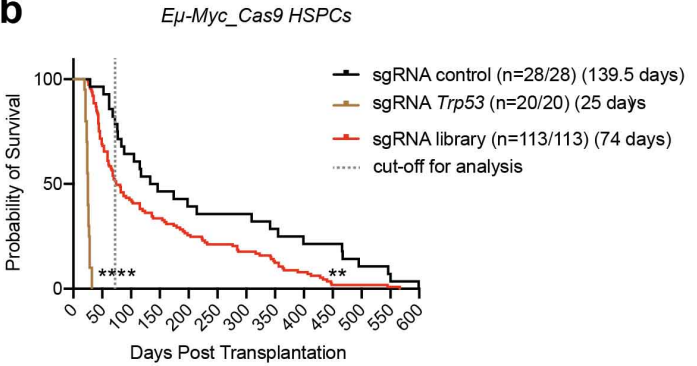

C

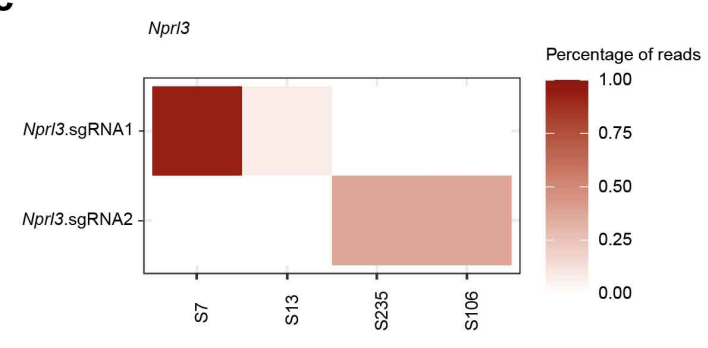

Figure 1. In vivo genome-wide CRISPR/Cas9 knockout screen identifies novel candidate tumour suppressors.

a, Schematic of experimental strategy for performing in vivo genome-wide screens to identify novel tumour suppressors. Foetal liver cells from E13.5 E $\mu$-Myc_Cas9 mice (C57BL/6-Ly5.2), a rich source of haematopoietic stem and progenitor cells (HSPCs), were transduced with lentiviruses containing sgRNAs targeting Trp53 (positive control), a negative control, or a whole genome sgRNA library ${ }^{15}$. The transduced foetal liver cells were injected i.v. into lethally irradiated recipient C57BL/6-Ly5.1 mice. Lymphoma bearing mice presented with enlarged spleen, lymph nodes and/or thymus. These tissues were harvested and cryopreserved. Genomic DNA was isolated from spleen cells and enriched sgRNAs identified by NGS. b, Kaplan-Meier survival curve showing tumour-free survival of mice reconstituted with $E \mu-M y c \_C a s 9$ HSPCs that had been lentivirally transduced with a sgRNA targeting Trp53 (positive control), the negative control sgRNA or a whole-genome sgRNA library. The dotted line represents cut-off for accelerated tumours for further analysis. $\mathrm{n}$ represents total number of reconstituted mice per cohort. Median survival is indicated in brackets. Log-rank (Mantel-Cox) statistical test for survival curve comparison, $* * P=0.0002$, $* * * * P<0.0001$. c, Heatmap representing Nprl3 sgRNAs enriched in accelerated tumours. Data are presented as proportion of reads assigned to sgRNA over all reads within a given tumour. $\mathrm{n}=4$ tumours where $\mathrm{Nprl} 3 \mathrm{sgRNAs}$ were enriched. 

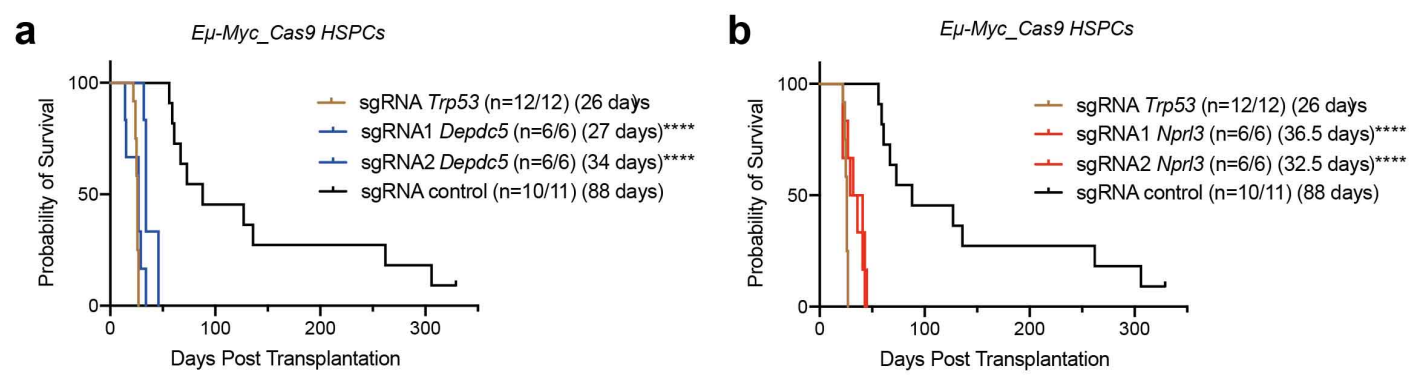

C

d

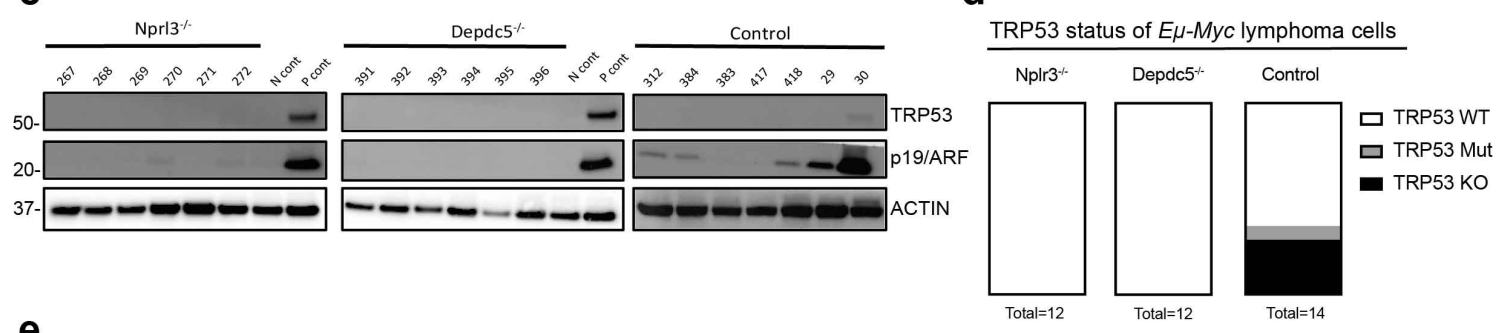

e

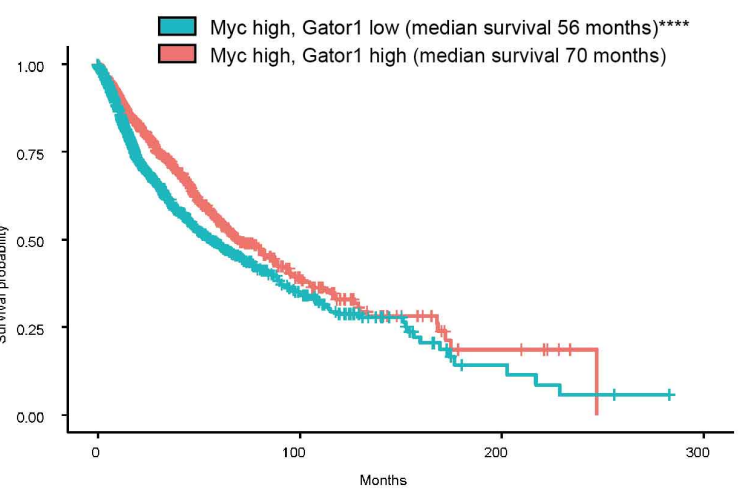

Figure 2. Validation and characterisation of the top candidate tumour suppressors Depdc5 and Nprl3.

Kaplan-Meier survival curves showing tumour-free survival of mice reconstituted with E13.5 E $\mu-M y c \_C a s 9$ foetal liver cells that had been transduced with a sgRNA targeting Trp53 (positive control), a negative control sgRNA or two independent sgRNAs each for targeting either Depdc5 a, or Nprl3 b. $\mathrm{n}$ represents the number of mice that developed lymphoma from a given cohort of reconstituted mice. Median survival is indicated in brackets. Log-rank (Mantel-Cox) test was used for comparison of mouse survival curves, $* * * * P<0.0001$. c, Representative Western blot analysis of TRP53 and p19/ARF levels to assess TRP53 status in primary control, DEPDC5-deficient or NPRL3-deficient $E \mu$ - $M y c$ lymphomas. Probing for ACTIN served as protein loading control. Protein size standards in $\mathrm{kDa}$ are indicated on the left-hand side. Lysates from Trp53 wt (N cont.) and Trp53 mutant (P cont.) E $\mu$-Myc lymphoma cells were run as 
controls. d, Proportion of control, DEPDC5-deficient or NPRL3-deficient $E \mu-M y c$ lymphomas that are Trp53 WT, Trp53 mutant (Mut) or Trp53 knockout (KO). The number of tumours analysed is indicated below the bars. e, Human TCGA Pan-Cancer analysis of $M Y C$ high expressing (top $25 \%$ expression) tumours, showing the difference in probability of survival between low GATOR1 component expression (bottom $25 \%$ expression, median survival 47 months) versus high GATOR1 component expression samples (top 25\% expression, median survival 70 months). $* * * * P<0.0001$, KaplanMeier statistical test.
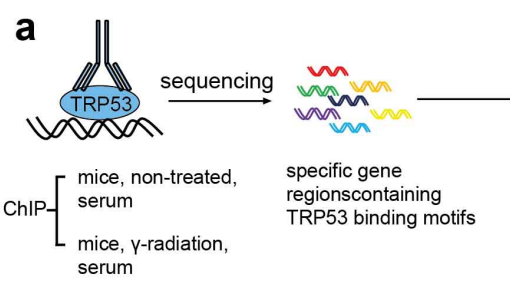

C

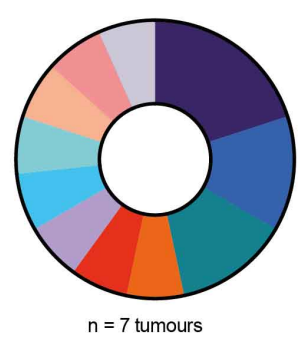

e

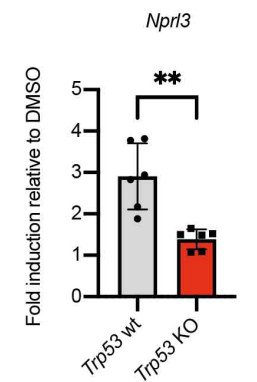

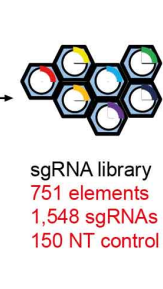

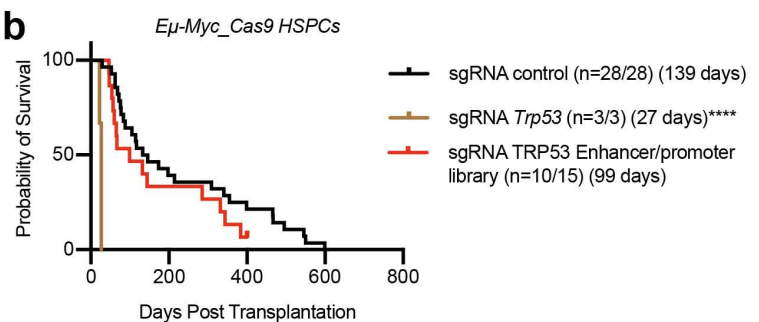

d

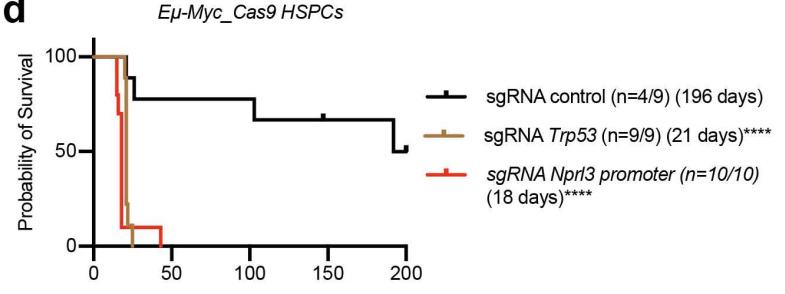

Days Post Transplantation

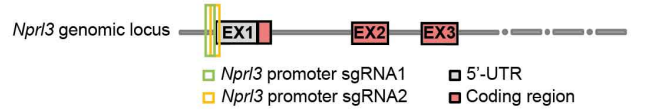

Figure 3. Targeting the TRP53 binding site in the $N p r l 3$ promoter accelerates $E \mu$ -

\section{Myc driven lymphoma}

a, Experimental strategy for constructing the focused TRP53 binding site

enhancer/promoter sgRNA library. The library contains 1,548 sgRNAs targeting the TRP53 binding sites in 751 TRP53 regulated genes and 150 non-targeting (NT) control sgRNAs. b, Kaplan-Meier survival curve showing tumour-free survival of mice 
reconstituted with E13.5 E $\mu-M y c \_C a s 9$ foetal liver cells that had been lentivirally transduced with a negative control sgRNA, a Trp53 specific sgRNA (positive control) or the focused TRP53 binding site enhancer/promoter sgRNA library. $\mathrm{n}$ represents the number of reconstituted mice per cohort with median survival indicated in brackets. Log-rank (Mantel-Cox) test for comparison of mice survival curves, $* * * * P<0.0001$. c, Pie graph presenting top enriched sgRNAs identified by NGS in the seven accelerated lymphomas from the screen of the TRP53 binding site enhancer/promoter library. In brackets, $\mathrm{n}$ represents the number of tumours that any sgRNA for a gene was identified in, sg represents the number of unique sgRNAs for a particular gene identified in accelerated tumours out of the number of sgRNAs for a gene in the library d, KaplanMeier survival curve showing tumour-free survival of mice reconstituted with E13.5 $E \mu-M y c \_$Cas9 foetal liver cells that had been lentivirally transduced with a negative control sgRNA, a Trp53 specific sgRNA (positive control) or one sgRNA targeting the TRP53 binding site in the $N p r l 3$ promoter. $\mathrm{n}$ represents the number of reconstituted mice per cohort. The median survival is indicated in brackets. Log-rank (Mantel-Cox) statistical test for survival curve comparison, $* * * * P<0.0001$. Schematic representation of the two sgRNAs targeting the Nprl3 genomic locus from the library: sgRNA1, which directly overlaps the TRP53 consensus binding sequence, was used in the validation experiment. e, Expression of Nprl3 and Cdkn1a/p21 mRNA in isogenic Trp53 wt or Trp53 knockout (KO) E $\mu$-Myc lymphoma cell lines treated for $6 \mathrm{~h}$ with $1 \mu \mathrm{g} / \mathrm{mL}$ etoposide (or DMSO as a negative control) in the presence of Q-VD-OPH (to prevent caspase mediated cell demolition) as determined by qRT-PCR analysis. Data were normalised to the $\mathrm{Hmbs}$ housekeeping gene expression and are presented as relative to untreated cells, mean \pm SD. $n=3$ cell lines, two independent experiments. Unpaired, two-tailed Student $t$-test, $* P<0.05, * * P<0.005$. 

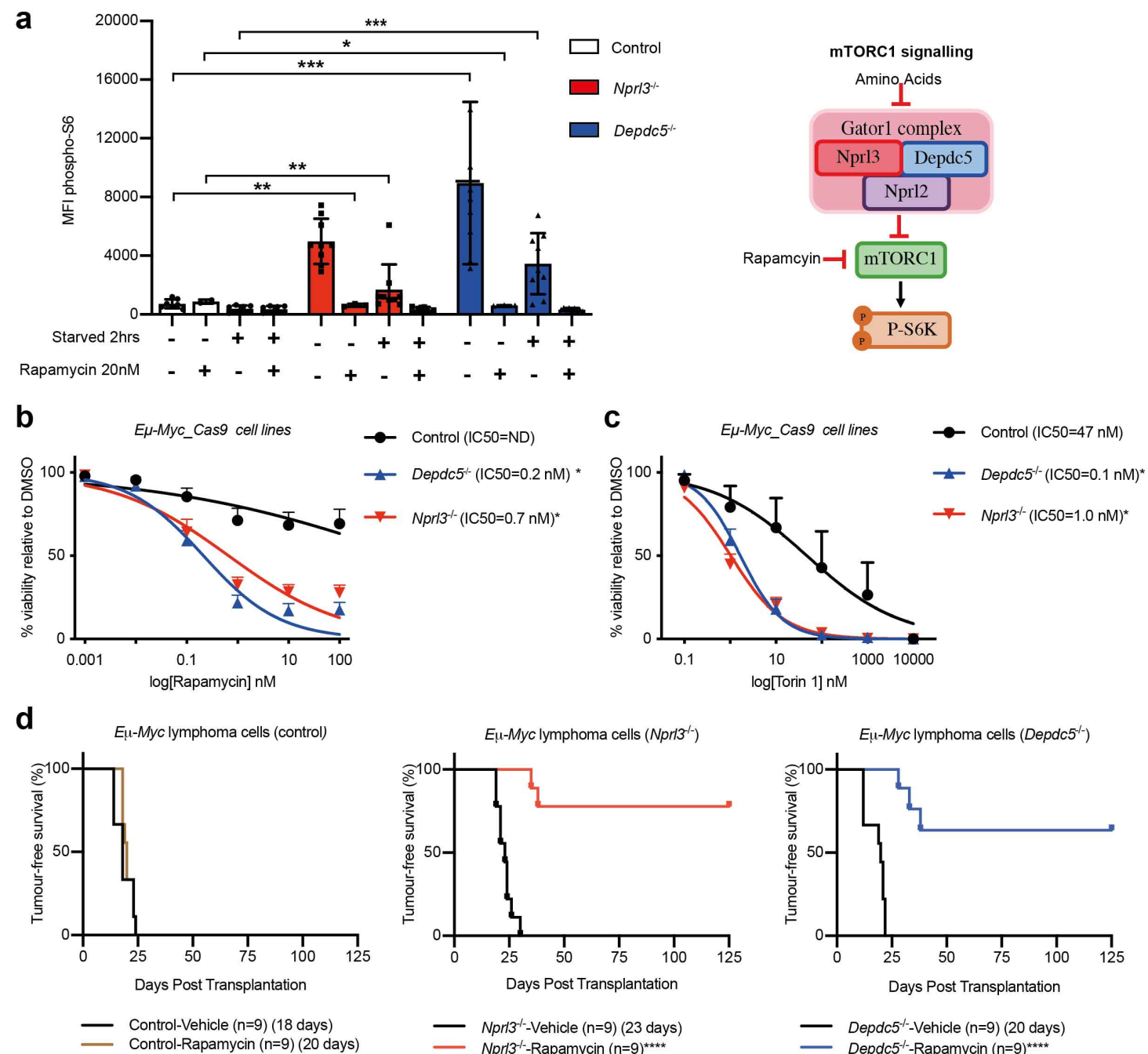

Figure 4. DEPDC5- and NPRL3-deficient $E \mu$-Myc lymphoma cells are addicted to mTOR signalling.

a, phospho-S6K (i.e. activated S6) protein expression as measured by intracellular flow cytometry (mean fluorescence intensity) in control as well as NPRL3-deficient or DEPDC5-deficient $E \mu-M y c$ lymphoma cell lines at steady state; steady state with rapamycin $(20 \mathrm{nM}) ; 2 \mathrm{~h}$ of starvation; and $2 \mathrm{~h}$ of starvation plus treatment with rapamycin $(20 \mathrm{nM})$ (left). Schematic of mTORC1 signalling pathway (right). $n=2-3$ cell lines per genotype across three replicate experiments. Non-parametric KruskalWallis test and uncorrected Dunn's test, ${ }^{*} P=0.0332, * * P=0.0021$, $* * * P=0.0002$, $* * * * P<0.0001$. Response curves of control, NPRL3-deficient or DEPDC5-deficient $E \mu$ Myc lymphoma cell lines treated with increasing doses of rapamycin $\mathbf{b}$, or torin1 $\mathbf{c}$. Cell viability was measured after $24 \mathrm{~h}$ of drug or vehicle treatment by staining with AnnexinV plus PI followed by flow cytometric analysis. AnnexinV PI double negative 
cells were deemed viable. $\mathrm{n}=3-6$ cell lines per lymphoma genotype across 3 technical replicates . Data are presented as mean \pm SEM, log transformed and fitting to non-linear regression; IC50 values in brackets. Student $t$-test with Welch's correction, $\mathrm{p}<0.05$. d, Kaplan-Meier survival curves of Rag-1 deficient mice that had been transplanted with $1 \times 10^{6}$ (left to right) control, NPRL3-deficient or DEPDC5-deficient E $\mu$-Myc lymphoma cell lines. Three days post transplantation, recipient mice were injected i.p. with daily doses of rapamycin $(8 \mathrm{mg} / \mathrm{kg}$ ) or vehicle (negative control) for five consecutive days and monitored for tumour free survival. $\mathrm{N}=9$ mice per treatment arm, with three cell lines per genotype each injected into three mice per. Log-rank (Mantel-Cox) statistical test for survival curve comparison, $* * * * P<0.0001$ 


\section{Materials and Methods}

\section{Lentiviral constructs and sgRNAs}

A positive sgRNA targeting Trp53 (5'-GGCAACTATGGCTTCCACCT; and a nontargeting (control) sgRNA targeting human-BIM 5'-GCCCAAGAGTTGCGGCGTAT were derived from the constitutive sgRNA FUGW expression vector previously described ${ }^{4}$. The genome-wide mouse lentiviral CRISPR sgRNA library used for in vivo screening experiment, YUSA v1, was a gift from Kosuke Yusa (Addgene \#50947) ${ }^{15}$. Two independent sgRNAs for in vivo validation experiments of candidate genes were obtained from the Merck CRISPR glycerol stock arrayed mouse sgRNA library available at WEHI (www.sigmaaldrich.com MSANGERG).

\section{Design, construction, and cloning of focused TRP53 binding site enhancer/promoter sgRNA library}

The TRP53 ChIP-seq data generated in Tonelli et al's study [PMID: 26372730] were downloaded from GEO database (GSM1828855, GSM1828856, GSM1828857 and GSM1828858). These data included an irradiated B cell sample, an irradiated non-B cell sample, a mock B cell sample and a mock non-B cell sample. ChIP-seq reads were mapped to the MGSCv37/mm9 reference genome using the Subread aligner [PMID: 23558742, 30783653]. Only uniquely mapped reads were retained.

Read mapping results were provided to the Homer program [PMID: 20513432] for peak calling. TRP53 binding peaks were called for irradiated B-cell and non-B-cell samples separately using an FDR cut-off of 1e-3, with the corresponding mock samples as control. Consensus binding motif sequences and corresponding position weight matrices (PWMs) were also obtained from Homer, based on the analysis of genomic sequences found within called peaks. The PWM contains the frequency of each nucleotide type (A, $\mathrm{T}, \mathrm{G}$ and $\mathrm{C}$ ) at each location of an enriched motif. The most enriched consensus motif sequence found for the two irradiated samples was TRP53 binding motif (CATGNNNNNNCATG).

We then ran Homer with the TRP53 PWM to search for exact binding sites of TRP53 in the called peaks. After obtaining all binding sites, we examined the presence of PAM 
(protospacer adjacent motif) sequence (NGG or $\mathrm{CCN}$ ) within these sites and their $6 \mathrm{bp}$ flanking regions. We only kept those binding sites that had at least one PAM sequence that had its associated cleavage site located within the $14 \mathrm{bp}$ binding sequence. The filtered binding sites from the two irradiated samples were then combined to generate a list of unique binding sites. These binding sites were then extended to both upstream and downstream directions by 50bp to create TRP53 binding regions of $114 \mathrm{bp}$ long, which were submitted to the CRISPR Design tool (http://crispr.mit.edu)[PMID: 23873081] for sgRNA design. We filtered the sgRNAs reported by CRISPR Design by requiring their target cleavage site be located within the TRP53 motif sequence. After this filtering, we removed those sgRNAs that had the identical sequence or had only $1 \mathrm{bp}$ difference between their sequences to create the final list of sgRNAs that were provided to Cellecta for sgRNA library construction.

sgRNAs targeting TRP53 binding regions in the gene promoters were identified by comparing sgRNA target locations against the promoter region of each gene, which was defined as a region including a $5 \mathrm{~kb}$ upstream sequence and a $2 \mathrm{~kb}$ downstream sequence relative to the transcriptional start site of the gene. If the target location of a sgRNA was not located in any promoter or gene body, then it was identified as a sgRNA targeting a TRP53 binding site in a gene enhancer region. The RefSeq mouse gene annotation (build 37.2) was used for this analysis.

\section{Lentivirus production}

Lentivirus was produced by transient transfection of HEK293T cells in $10 \mathrm{~cm}^{2}$ dishes with $10 \mu \mathrm{g}$ of vector DNA and packaging lentiviral constructs pMDL ( $5 \mu \mathrm{g}), \mathrm{RSV}(2.5$ $\mu \mathrm{g})$, and $\mathrm{pENV}(5 \mu \mathrm{g})$ by the standard calcium phosphate precipitation method previously described ${ }^{32}$. Viral supernatants were collected $48-72 \mathrm{~h}$ after transfection and passed through a $0.45 \mu \mathrm{M}$ filter prior to transduction.

\section{Animal experiments}

The care and use of experimental mice were conducted according to guidelines of The Walter and Eliza Hall Institute Animal Ethics Committee. C57BL/6-Ly5.2+, C57BL/6Ly5.1+, Rag1/J mice were obtained from the Walter and Eliza Hall Institute breeding facility (Kew, Victoria, Australia). $E \mu-M y c$ transgenic ${ }^{11}$ and Cas9 transgenic mice (a 
gift from K.Rajewsky) ${ }^{33}$ mice were maintained on a C57BL/6-Ly5.2 background. For haematopoietic reconstitution, embryonic day E13.5 foetal liver cells (a rich source of HSPCs) were collected from $E \mu-M y c^{\mathrm{T} /+}{ }_{-} \mathrm{Cas} 9^{\mathrm{KI} /+}$ doubly transgenic mice on a C57BL/6-Ly5.2 background and cultured in alpha-minimum essential medium ( $\alpha$ MEM) GlutaMAX (Gibco) supplemented with 10\% FCS (foetal calf serum; Gibco), 1 $\mathrm{mM}$ L-glutamine, $1 \mathrm{mM}$ sodium pyruvate, $100 \mathrm{U} / \mathrm{mL}$ penicillin, $100 \mu \mathrm{g} / \mathrm{mL}$ streptomycin, $10 \mathrm{mM}$ HEPES, $50 \mu \mathrm{M} \beta$-mercaptoethanol and recombinant cytokines. The cytokines IL-6 (10 ng/mL), mouse stem cell factor (100 ng/mL), thrombopoietin (50 ng/mL), and FLT-3 ligand (10 ng/mL) were kindly provided by Dr. Jian-Guo Zhang (WEHI). HSPCs were cultured for $48 \mathrm{~h}$ for lentiviral infection. Infections for the generation of viral supernatants described above were conducted in a 12-well non-tissue culture treated plate that had been coated overnight with retronectin solution $(32 \mu \mathrm{g} / \mathrm{mL}$ in PBS) at $4{ }^{\circ} \mathrm{C}$ followed by blocking with $2 \%$ bovine serum albumin solution (\#A1595 Sigma-Aldrich) in PBS at $37^{\circ} \mathrm{C}$ for 30 min prior to coating with viral supernatant. Viral particles supplemented with $8 \mu \mathrm{g} / \mathrm{mL}$ polybrene, were centrifuged onto the retronectin plates at 3,000 rpm for $2 \mathrm{~h}$. HSPCs were then added to wells and incubated for $24 \mathrm{~h}$. HSPCs transduced with vectors encoding sgRNAs were collected, washed in PBS, and then injected into lethally irradiated (two doses of 5.5 Gy, $4 \mathrm{~h}$ apart) C57BL/6-Ly5.1 mice. These transplanted mice were carefully monitored for illness by lymph node/spleen palpitation and overall appearance. Survival time was defined as the time from transplantation until the animal was sick and had to be euthanised for ethical reasons. Peripheral blood was collected and analysed using an ADVIA haematology analyser (Bayer) and haematopoietic tissues (spleen, lymph nodes, thymus, bone marrow) were collected for downstream analysis and freezing.

For in vivo drug treatement, $1 \times 10^{6} E \mu-M y c$ lymphoma (Ly5.2) cells resuspended in 200 $\mu \mathrm{L}$ sterile PBS were injected into 7 12week old sex-matched recipient Rag1-deficient mice by intravenous (i.v.) tail vein injection. After three days, mice were treated for five consecutive days with vehicle or $8 \mathrm{mg} / \mathrm{kg}$ rapamycin by intraperitoneal (i.p.) injection. Rapamycin (LC laboratories) was first dissolved in $100 \%$ ethanol, stored at $-20^{\circ} \mathrm{C}$ and further diluted in vehicle, 5.2\% PEG 400 and 5.2\% Tween 80, immediately before use. Survival time was defined as the time from lymphoma cell line injection until mice were sick and had to be euthanised according to our animal ethics guidelines by an experienced animal technician. 


\section{Genotyping}

Mouse genotypes were determined by PCR analysis of DNA derived from earclips or embryonic tail isolated using direct tail lysis buffer (Viagen Biotech) and Proteinase K. Mastermix was prepared with GoTaq green (Promega, M7123) containing primers (final concentration $0.5 \mathrm{pmol} / \mu \mathrm{L}$ ) with the addition of $1 \mu \mathrm{L}$ total DNA. PCR cycling conditions were: $94 \mathrm{C} 4$ min followed by 30 cycles ( $94 \mathrm{C}$ for $40 \mathrm{sec}, 55 \mathrm{C}$ for $30 \mathrm{sec}, 72$ $\mathrm{C}$ for $60 \mathrm{sec}$ ) and finally $72 \mathrm{C}$ for $5 \mathrm{~min}$. PCR products were separated by gel electrophoresis on 2\% DNA grade agarose (Bioline) in TAE buffer(40 mM Tris Acetate, $1 \mathrm{mM}$ EDTA pH 8.0) containing ethidium bromide $(0.2 \mu \mathrm{g} / \mathrm{mL}$, Sigma $)$ and imaged on GelDoc DOCTM XR+ Gel Documentation system (Bio-Rad). PCR oligonucleotide primers (Integrated DNA Technologies) and expected products were as follows:

E्म-Myc: $\sim 900 \mathrm{bp}$

myc-1: 5' -CAGCTGGCGTAATAGCGAAGAG

myc-2: 5' -CTGTGACTGGTGAGTACTCAACC

\section{Next generation sequencing}

Genomic DNA was isolated from lymphoma cells using DNeasy Blood and Tissue Kit (QIAGEN) according to the manufacturer's instructions. For each lymphoma, integrated sgRNAs were amplified from 100 ng of genomic DNA using GoTaq Green Master Mix (Promega) and indexing primers with unique overhangs ${ }^{16}$. Amplicons were pooled, cleaned up using Ampure XP beads (Beckman Coulter) and sequenced on Illumina Nextseq or Miseq platform. Enriched sgRNAs per tumour were calculated by number of reads mapping to each sgRNA in the library as a proportion of the total number of reads within a tumour sample.

\section{Cell culture and in vitro drug treatment assays}

HEK293T cells were cultured in DMEM medium supplemented with $10 \%$ heat inactivated (HI)-FCS. Prior to virus production, HEK293Ts were cultured in DME Glutamax (Gibco) supplemented with 10\% FCS and 25mM HEPES. E $\mu$-Myc lymphoma cell lines derived from tumours of sick mice were maintained in FMA medium as previously described ${ }^{34}$. To assess drug sensitivity of $E \mu-M y c$ lymphoma 
cells, $5 \times 10^{4}$ cells were plated in triplicate into 96-well flat-bottom plates. Cells were treated with rapamycin (Cell Signalling Technology, \#9904), torin1 (Cell Signalling Technology, \#14379), S63845 (Active Biochem, \#6044), Nutlin-3A (Cayman Chemical, \#18585) or Etoposide (Ebewe Pharmaceuticals Ltd, A-4866). Cell viability was assessed after $24 \mathrm{~h}$ by staining cells with $2 \mu \mathrm{g} / \mathrm{mL}$ propidium iodide (PI) and AnnexinV conjugated to Alexa Fluor 647 (WEHI) using the Fortessax20 flow cytometer (Becton Dickinson), 10,000 cells were recorded per sample. Data were analysed using FlowJo analysis software. Cell viability was normalised to control cells treated with an equivalent volume of vehicle, either dimethylsulfide (DMSO), water or $100 \%$ ethanol.

To assess phospho-S6 levels, $E \mu-M y c$ lymphoma cells were washed in PBS then resuspended in either FMA or Hanks Balanced Salt Solution (HBSS), for starvation condition, supplemented with or without $20 \mathrm{nM}$ rapamycin and incubated for $2 \mathrm{~h}$ at $37^{\circ} \mathrm{C}$ in $10 \% \mathrm{CO}_{2}$. Cells were spun down, washed in PBS and either protein was isolated for Western blotting or intracellular flow cytometry was performed. To assess TRP53 target genes, three isogenic Trp53 wt or Trp53 KO E $\mu$-Myc lymphoma cell lines were generated using CRISPR/Cas9. 1.2 × $10^{6} \mathrm{E} \mu$-Myc lymphoma cells were plated in FMA medium supplemented with $25 \mu \mathrm{M}$ Q-VD-OPH (broad spectrum caspase inhibitor, MP Biomedicals) and subsequently etoposide $(1 \mu \mathrm{g} / \mathrm{mL})$ or an equal volume of DMSO was added and cells were harvested at $6 \mathrm{~h}$ of treatment for gene expression analysis.

\section{Flow cytometric analysis}

Immunophenotyping of lymphomas was performed on single cell suspensions of primary lymphoma tissue from sick mice or $E \mu-M y c$ lymphoma derived cell lines stained with fluorochrome-conjugated antibodies against B220 (RA3-6B2), IgM (5.1), IgD (11-26C), T cell antigen receptor beta (TCR $\beta$, H57-597) and CD19 (ID3) in PBS supplemented with 5\% FCS and Fc block (2.4G2 supernatant, WEHI). Donor derived cells were identified as Cas9-eGFP positive and sgRNA CFP or BFP positive. Propidium iodide (PI), final concentration of $2 \mu \mathrm{g} / \mathrm{mL}$, was used for excluding dead cells during analysis on a Fortessax20 flow cytometer (Becton Dickinson). Expression of phospho-S6K was assessed in $E \mu-M y c$ lymphoma cells following the above described starvation. $1 \times 10^{5}$ cells were harvested, washed in PBS and stained with green 
fixable viability dye (Thermo Fisher Scientific). Cells were fixed for $1 \mathrm{~h}$ using eBioscience $^{\mathrm{TM}}$ Foxp3/Transcription Factor Staining Buffer Set (Thermo Fisher Scientific) and stained with an antibody against phospho-S6 conjugated to Alexa Fluor 647 (Cell Signalling Technology, \#4851) according to the manufacturer's instructions and cells were then analysed on a LSR IIC flow cytometer (Becton Dickinson). Data were analysed using FlowJo analysis software and presented as geometric mean fluorescence intensity (MFI).

\section{Western blotting}

Total protein extracts were prepared from $E \mu-M y c$ lymphoma cell lines and primary lymphomas by lysis in RIPA buffer (50 mM Tris-HCl, $150 \mathrm{mM} \mathrm{NaCl}, 1 \% \mathrm{NP}-40,0.5 \%$ DOC, $0.1 \%$ SDS) containing complete protease inhibitor cocktail (Roche) and phosphatase inhibitor cocktail (PhosphoSTOP ${ }^{\mathrm{TM}}$, Roche). Protein concentration was quantitated by the Bradford assay (Bio-Rad), between 10 to $30 \mu \mathrm{g}$ of protein were prepared in Laemmli buffer boiled for 5 min and separated by SDS-PAGE on NuPAGE 10\% Bis-Tris $1.5 \mathrm{~mm}$ gels (Life Technologies). Proteins were then transferred onto nitrocellulose membranes (Life Technologies) using the iBlot membrane transfer system (Thermo Fisher Scientific). Membranes were incubated with the primary and then the HRP-conjugated secondary antibodies detailed in Extended data table 2, diluted in PBS containing $10 \%$ bovine serum albumin with $0.1 \%$ Tween 20 . Luminata Forte Western horseradish peroxidase (HRP) substrate (Merck Millipore) was used for developing the signal and membranes were imaged and analysed using the ChemiDoc Imaging System with ImageLab software (Bio-Rad).

\section{qRT-PCR analysis}

Total RNA was extracted using QIAzol reagent (Qiagen) and complementary DNA was synthesized using SuperScript First Strand Synthesis SuperMix (Thermo Fisher Scientific), according to respective manufacturer's instructions. Quantitative reversetranscription polymerase chain reaction (qRT-PCR) analysis was performed on the ViiA7 Real-time PCR system (Thermo Fisher Scientific) using TaqMan Gene Expression Assays (Applied Biosystems) in triplicate as detailed in Extended data table 3. Data were analysed using QuantStudio software (Thermo Fisher Scientific).

Messenger RNA (mRNA) levels were normalised to the expression of the housekeeping 
gene Hmbs. All qPCR data were analysed using $2^{-\Delta \Delta \mathrm{CT}}$ method and presented relative to untreated sample.

\section{Human TCGA Pan-Cancer Analysis}

TCGA pan-cancer RSEM batch normalised mRNA expressions was downloaded from the cBioPortal ${ }^{35,36}$ on July $30^{\text {th }}, 2021$. The R statistical software (version 4.1.2) was used to conduct survival analysis. The Kaplan-Meier survival statistics were computed using R package 'survival' (version 3.2-13) and plotted using R package 'survminer' (version 0.4.9).

\section{Statistical analysis}

GraphPad Prism software (Version 8) was used to plot Kaplan-Meier survival curves of reconstituted mice. For comparing two groups, Student's $t$-test was used, and for comparing more than two groups multiple t-test or two-way ANOVA were performed, unless otherwise stated in the figure legends. Data are presented as mean \pm standard deviation (SD), unless otherwise stated in figure legends. Significance is measured where $P$ value is less than 0.05 , ns $=$ no significant difference. For the exploratory analysis of the sgRNA counts, heatmaps and pie charts were plotted using R package 'ggplot' (version 2_3.3.5).

\section{Acknowledgements}

The authors thank all members of the Blood Cells and Blood Cancer Division at WEHI for their support, advice, and sharing of reagents; Giovanni Siciliano and other Bioservices staff for technical assistance with the in vivo experiments and animal husbandry; A Aeslop and the Screening lab at WEHI for the Glycerol stock sgRNAs for target validation. This work was supported by grants and fellowships from the Australian National Health and Medical Research Council (NHMRC) (Project Grants 1159658,1186575 and 1145728 to MJH, 1143105 to MJH and AS, Ideas Grants 2002618 and 2001201 to GLK, Program Grant 1113133 to AS and Fellowships 1020363 to AS, 1156095 to MJH), the Leukemia and Lymphoma Society of America (LLS SCOR 701518 to AS, GLK, MJH), the Cancer Council of Victoria (project grant 1147328 and 2021 Grant In Aid to MJH, 1052309 to AS ,1147328 to GLK and Venture Grant to MJH and AS), Victorian Cancer Agency (MCRF Fellowship 17028 to GLK, Leukaemia 
Foundation of Australia (grant to AS and GLK), Phenomics Australia (to AJK and MJH) the estate of Anthony (Toni) Redstone OAM (AS and GLK), the Craig Perkins Cancer Research Foundation (GLK), the Dyson Bequest (GLK), the Harry Secomb Trust (GLK) and the University of Melbourne Research Training scholarship (MAP). This work was made possible by operational infrastructure grants through the Australian Government Independent Research Institute Infrastructure Support Scheme (361646 and 9000220) and the Victorian State Government Operational Infrastructure Support Program.

\section{Author contributions}

M.J.H, and A.S, conceived and designed the research. S.M. M.A.P., Y.D., G.H., performed the experiments; G.G., performed bioinformatic and statistical analysis. Y.L. and W.S. performed bioinformatic analysis and designed TRP53 enhancer/promoter sgRNA library; S.W. ran NGS; G.P. and A.W. provided reagents; S.M., M.A.P., Y.D., M.J.H, and A.S. analysed and interpreted results; S.M. and M.A.P., M.J.H. and A.S. drafted, edited and revised the manuscript with help from all the authors.

\section{Competing interests}

The authors declare no conflicts of interest with respect to this work.

\section{Additional information}

Extended data and information is available for this paper. Correspondence and requests for materials should be addressed to Marco Herold herold@wehi.edu.au. 